\title{
Primate model to study reactivation of TB associated with retroviral infection
}

\author{
Stephen D Lawn ${ }^{\dagger} \&$ Robert J Wilkinson $n^{1,2,3}$ \\ Institute of Infectious Disease \& Molecular Medicine, University of Cape Town, South Africa \\ 2Division of Medicine, Imperial College London, W2 1PG, UK \\ ${ }^{3}$ MRC National Institute for Medical Research, Mill Hill, London, UK \\ ${ }^{\dagger}$ Author for correspondence: The Desmond Tutu HIV Centre, Institute of Infectious Disease \& Molecular \\ Medicine, Faculty of Health Sciences, University of Cape Town, Cape Town, South Africa and Clinical \\ Research Unit, Department of Infectious and Tropical Diseases, London School of Hygiene \& Tropical \\ Medicine, London, UK w Tel.: +44 270216506970 m Fax: +44 270216506963 m stevelawn@yahoo.co.uk
}

Evaluation of: Diedrich CR, Mattila JT, Klein E et al:: Reactivation of latent tuberculosis in cynomolgus macaques infected with SIV is associated with early peripheral T cell depletion and not virus load. PLOS ONE 5(3), e9611 (2010). This work describes the effect of simian immunodeficiency virus (SIV)mac251 infection on clinically inapparent (latent) TB infection in Cynomolgus macaques (Macaca fascicularis). SIV and TB coinfected animals were compared with those with SIV infection alone and with SIV-uninfected animals with latent or active TB. All seven animals with latent TB reactivated within 11 months of SIV infection. Animals were divided into those that reactivated less than 17 weeks post-SIV infection and those that reactivated more than 26 weeks post-SIV infection. Reactivation was independent of viral load but related to depletion of T cells during acute SIV infection. Granulomas from animals with reactivated TB demonstrated a spectrum of distinct pathological features consistent with chronic inflammation. It is of interest to relate these findings to the poorly defined mechanisms by which the risk of human TB increases soon after acquisition of HIV-1 infection. Furthermore, an animal model that faithfully recreates HIV-associated susceptibility to TB would be of great utility when considering novel immune and chemotherapeutic options for HIV and TB coinfected individuals.

If current trends continue, the Millennium Development Goal (MDG) targets for TB control will not be achieved by 2015 [101]. One of the key obstacles to progress is the HIV-associated TB (HIV-TB) epidemic, with approximately 1.3 million new cases and almost half a million deaths in $2008[1,102]$. While this, in part, reflects a major deficiency in the implementation of the public health response in the countries hardest hit [2], this is also an indication of the complexity of the challenge. A robust research agenda is needed to provide new insights into the copathogenesis of these diseases, leading to development of new tools and interventions.

Advanced preclinical evaluation of novel anti$\mathrm{TB}$ drugs and novel vaccines against $\mathrm{TB}$ and HIV-associated TB requires an animal model that closely recreates the pathological features of human TB in a way that no small animal model can achieve [3,4]. Over the last 10 years JoAnne Flynn and colleagues have developed low-dose airborne infection of Cynomolgus macaques with Mycobacterium tuberculosis for this purpose, and the histopathological spectrum of lesions observed is close to that of human TB [3,5-7]. Of particular interest is that low-dose infection does not become clinically apparent in approximately half of the animals but these exhibit clear pathological, immunological and occasional microbiological evidence of latent TB. The model has been used to better understand reactivation of $\mathrm{TB}$ resulting from the therapeutic use of anti-TNF antibodies, similar to what occurs in humans $[8]$, and also for evaluation of new therapeutic and vaccine strategies.

Given that HIV-1 infection is the strongest known risk factor for the reactivation of TB in humans, it was obviously tempting to employ this latent TB model to see if the clinical circumstances can be recreated and thereby more easily elucidate the underlying immune mechanisms. Therefore, seven macaques with latent $\mathrm{TB}$ were infected with simian immunodeficiency virus (SIV)mac251 and compared with four macaques with SIV infection alone and with a variable number of macaques with latent or active $\mathrm{TB}$ in the absence of SIV infection. The latter animals were not subject to the same experimental protocol, instead data were derived from previous experiments conducted in similar circumstances in the same laboratory [9].
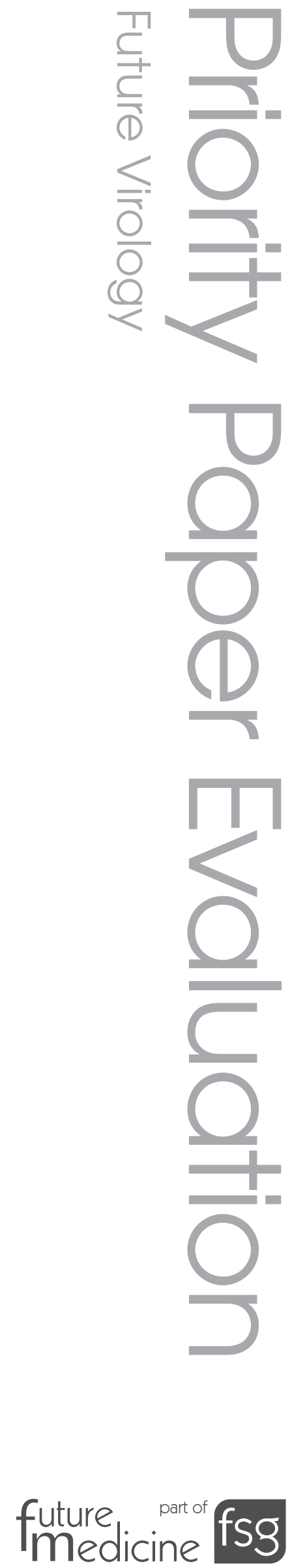
All the SIVmac251-infected animals developed reactivation $\mathrm{TB}$ and the rate at which they did so was related to the degree of initial T-cell depletion but not viral load during the acute phase of SIV infection. The spectrum of pathological lesions varied according to the time to reactivation, with those animals that reactivated late having a tendency to less extensive disease and evidence that some lesions may have been healing. Lesions contained fewer $\mathrm{T}$ cells than those from SIV-uninfected animals with active TB. Therefore, the authors suggest that it is the acute phase of SIV infection that perturbs immune containment of $\mathrm{TB}$, perhaps via the deletion of critical T-cell phenotypes, which sets up the path to inevitable reactivation at some point.

An issue that may attract comment is that, following acute infection, the viral set point in plasma was quite low. This is not the case in humans infected with HIV-1 or in Rhesus macaques infected with SIV. However, the authors do demonstrate much higher SIVmac251 viral load in tissues. Interestingly, they also report on two Cynomolgus macaques infected with a different SIV-HIV chimera SHIV89.6. These macaques had profound progressive depletion of peripheral CD4 cell numbers but not of mucosal CD4 cell numbers and did not develop reactivation TB. The authors make the point that peripheral CD 4 cell numbers are imperfect indicators of TB risk and hypothesize that cell numbers within mucosae may be a critical factor.

Another relative weakness of this work is the small number of animals studied, which limited the power of analyses. It must be noted that the findings, although extensive, are preliminary and that the cost and ethics of experimentation on nonhuman primates do not permit large sample sizes. Another weakness is the use of historical controls, although these were the same species researched in the same laboratory, infected and analyzed in exactly the same way by the same investigators.

There are several other interesting points when considering this model in the context of human HIV-TB interaction. First, all of the SIV-infected macaques developed reactivation $\mathrm{TB}$, which, despite the increased risk conferred by HIV-1, does not occur in all humans. The data from animals with late reactivation suggest that an initial acute SIV-mediated perturbation of immune containment followed by an attempt to regain immune control and some healing may be most relevant. The potential for loss of crucial mucosal T-cell subsets early in infection is corroborated by findings in humans. In bronchoalveolar lavage of healthy HIV-infected patients exposed to TB the frequencies of cells expressing IFN- $\gamma$ or TNF- $\alpha$, as well as polyfunctional cells (coexpressing IFN- $\gamma$, TNF- $\alpha$ and IL-2), were lower in HIV-1infected persons than in uninfected controls [10]. Polyfunctional cells associate experimentally with vaccine-induced protection against $\mathrm{TB}$ [11].

Interestingly, while it is recognized that sites of TB disease in humans are associated with increased HIV-1 replication [12,13], the authors report that sites of active TB infection were not associated with viral replication in their model. The authors acknowledge that the granuloma might well provide a permissive environment for retroviral replication but could not correlate viral load with $M$. tuberculosis burden in individual granulomas.

What insights are provided by these animal model data into the epidemiology and control of human HIV-TB epidemic? A key observation originally made among South African miners is that following HIV seroconversion, the risk of TB increases two- to three-fold within the first $1-2$ years of infection $[14,15]$ and continues to rise as CD 4 cell counts decrease [16-18]. The very early and marked increase in TB risk following HIV acquisition is striking and differs from many other opportunistic infections [16]. This suggests an early impact of HIV on antimycobacterial immune responses, which is consistent with the primate model findings.

HIV-infected patients with positive tuberculin skin tests have a substantially higher risk of $\mathrm{TB}$ than those with a negative skin test [18,19], which strongly implicates reactivation of latent $\mathrm{TB}$ as an important mechanism underlying increased TB risk following HIV infection. In the highest TB burden communities in South Africa, TB transmission rates are so high during childhood and adolescence that the majority of individuals who acquire HIV infection during early adulthood already have preceding latent TB infection [20]. Following HIV acquisition, early increases in risk of reactivation TB are consistent with the extraordinarily high TB risk among young adults in such communities [21].

Use of antiretroviral therapy (ART) is associated with a $54-92 \%$ reduction in TB incidence rates in treatment cohorts as functional antimycobacterial immune responses are restored [22,23]. Individual risk of TB during ART is directly related to changing absolute CD4 cell counts, with an almost tenfold difference in adjusted rates comparing patients with counts less than 100 cells $/ \mu l$ and more than 500 cells $/ \mu 1$ [24]. However, even among patients 
who achieve blood CD4 cell counts over 500 cells $/ \mu$ l, the risk remains approximately twofold higher than that of HIV-uninfected people living in the same community and similar to the risk that was present after HIV seroconversion. This supports the hypothesis that early devastating consequences of HIV acquisition lead to a persistent and irreversible defect in functional antimycobacterial immunity [23]. Future studies using the primate model may be able to elucidate this defect.

The early consequences of HIV infection on host immune control of $M$. tuberculosis during HIV-seroconversion may have implications for the timing of ART initiation. In very high TB prevalence settings, very early initiation of ART may be beneficial with regard to preservation of antimycobacterial immune responses and may be a further benefit that can be derived from the 'test-and-treat' strategy in which it has been proposed that individuals be tested annually and those found to be positive should immediately start ART regardless of CD4 cell count [25].

If there is a quantitative T-cell defect in TB granulomas in HIV-infected people, could increasing the number of vaccinations potentially be an approach to boost such numbers? Although it may seem counterintuitive to expect a vaccine dependent on T-cell-mediated immunity to be effective in HIV-infected persons, it has recently been shown that multiple doses of Mycobacterium vaccae administered to HIV-infected adults was safe and associated with some protection against microbiologically proven TB [26].

The early increase in TB risk following HIV seroconversion indicates that TB preventive interventions, such as isoniazid monotherapy, are required from the earliest stages of the HIV disease course. The benefit of isoniazid preventive therapy in HIV-1 infected persons appears to be short lived and restricted to those who are tuberculin skin test positive [27]. However, many questions remain unanswered. Is there a relationship between immune competence and the efficacy of this therapy, or does isoniazid only impact subpopulations of $\mathrm{TB}$ that are actively replicating without eradicating forms that may resume active replication once the therapy is withdrawn? Would multiple drugs targeting multiple bacillary processes be more effective? The trend of evidence is in favor of shorter courses of the combination of rifampicin and isoniazid or rifampicin, pyrazinamide and isoniazid, as these are as effective as isoniazid alone [28]. However, these regimens tend not to be used owing to a higher frequency of adverse events in HIV-uninfected persons from low-risk environments in whom the risk-benefit ratio is substantially different [29]. Drugs specific for latent $\mathrm{TB}$ would be an advantage and a relevant primate model to evaluate such drugs would be greatly advantageous.

Current thinking questions the longstanding paradigm of human infection with $M$. tuberculosis existing in distinct dichotomous forms of latent infection versus active disease [30]. The existence of a spectrum of infection/disease associated with rising mycobacterial numbers and changing immune phenotype appears more likely. Such a spectrum may be profoundly affected by HIV coinfection and this could be studied within the primate model.

In this regard, it is interesting to note that bronchoalveolar lavage fluid from some animals tested culture positive to $M$. tuberculosis while the extent of disease was still low. It is well recognized that culture-positive TB can occur in the presence of minimal symptoms and a normal chest radiograph in HIV-infected humans [31-33]. The assumption that such persons would inexorably go on to develop symptomatic disease cannot, for obvious reasons, be tested in a natural history study. However, the evidence of healing TB lesions in some SIVinfected macaques potentially suggest an alternative scenario in which HIV-infected persons with latent TB and relatively well-preserved immune function could be intermittently infectious. This would be important with regard to TB control.

\section{Future perspective}

It is now approximately 30 years since two epidemics caused by an ancient pathogen (M. tuberculosis) and a new one (HIV-1) became inseparably intertwined, with devastating consequences for public health worldwide. In contrast to HIV infection, for which rapid and highly sensitive and specific point-of-care diagnostic assays are available, correlation between disease progression and treatment response are easily measured and a raft of potent multidrug treatment combinations are available, the control of TB (and of HIV-TB in particular) remains sorely hampered. Diagnostics function poorly, drug treatment regimens are very limited and preventive interventions lack simplicity and efficacy. During the next 5 years, there is a pressing need to much more boldly implement interventions that are known to work [2]. However, development of newer interventions requires a vigorous 
TB research agenda that includes basic and preclinical studies. To date, progress on basic research into the copathogenesis of HIV and TB has been hampered by a number of factors, including the lack of an animal model. This primate model appears to be the first for latent TB in which the immunological and microbiological responses to retroviral infection can be directly investigated. While new TB diagnostics that have good performance characteristics in HIV-coinfected patients are now becoming available, the prospects for new TB drugs, specific agents that can be restricted to treatment of latent infection and a new TB vaccine, remain far in the future. The potential role of this primate model in advancing this HIV-TB research agenda remains to be seen.

\footnotetext{
Financial \& competing interests disclosure Both authors are funded by the Wellcome Trust (grants 084323 and 088316), London, UK. The authors have no other relevant affiliations or financial involvement with any organization or entity with a financial interest in or financial conflict with the subject matter or materials discussed in the manuscript apart from those disclosed.

No writing assistance was utilized in the production of this manuscript.
}

\section{Executive summary}

\section{Objectives of this study}

- To develop a novel primate model of HIV-induced reactivation TB.

- To examine the immunological, microbiological and virological changes in peripheral blood and tissues associated with reactivation TB.

\section{Methods}

- Cynomolgus macaques (Macaca fascicularis) infected with simian immunodeficiency virus (SIV) strain SIVmac251 were infected with the Erdmann strain of Mycobacterium tuberculosis using a low-dose airborne exposure.

- Animals were studied longitudinally until active TB was evident, at which point animals were euthanized and underwent necropsy.

- Animals coinfected with SIV and TB were compared with those with SIV infection alone and with SIV-uninfected animals with latent or active TB.

\section{Results}

- All seven animals with latent TB reactivated within 11 months of SIV infection. Animals were divided into those that reactivated less than 17 weeks post-SIV infection or those that reactivated more than 26 weeks post-SIV infection.

- Reactivation was independent of viral load but related to depletion of T cells during acute SIV infection. Animals with early reactivation had fewer T cells in peripheral blood and airways than animals with later reactivation.

\section{Conclusion}

- The initial T-cell depletion during acute retroviral infection may strongly influence subsequent risk of TB reactivation.

\section{Bibliography}

Papers of special note have been highlighted as:

- of interest

-. of considerable interest

1. Lawn SD, Churchyard G: Epidemiology of HIV-associated tuberculosis. Curr. Opin. HIV AIDS 4, 325-333 (2009).

2. Harries AD, Zachariah R, Corbett EL et al:: Confronting the HIV-associated tuberculosis epidemic - when will we act? Lancet 375 , 1906-1919 (2010).

3. Flynn JL, Capuano SV, Croix D et al:: Non-human primates: a model for tuberculosis research. Tuberculosis 83, 116-118 (2003).

4. Orme IM: The mouse as a useful model of tuberculosis. Tuberculosis 83, 112-115 (2003).

5. Capuano SV 3rd, Croix DA, Pawar S et al.: Experimental Mycobacterium tuberculosis infection of cynomolgus macaques closely resembles the various manifestations of human M. tuberculosis infection. Infect. Immun. 71, 5831-5844 (2003).
-. Description of the Cynomolgus macaque model of $\mathrm{TB}$, which shows strong similarities to infection and disease in humans.

6. Lin PL, Pawar S, Myers A et al.: Early events in Mycobacterium tuberculosis infection in cynomolgus macaques. Infect. Immun. 74, 3790-3803 (2006).

- Description of the clinical, microbiological and immunological events occurring during the initial weeks following infection of Cynomolgus macques with Mycobacterium tuberculosis.

7. Lin PL, Rodgers M, Smith L et al:: Quantitative comparison of active and latent tuberculosis in the cynomolgus macaque model. Infect. Immun. 77, 4631-4642 (2009).

- Detailed description of the spectrum of latent infection and active disease caused by Mycobacterium tuberculosis in Cynomolgus macaques.
8. Lin PL, Myers A, Smith L et al.: Tumor necrosis factor neutralization results in disseminated disease in acute and latent Mycobacterium tuberculosis infection with normal granuloma structure in a Cynomolgus macaque model. Arthritis Rheum. 62, 340-350 (2010).

9. Diedrich CR, Mattila JT, Klein E et al.: Reactivation of latent tuberculosis in cynomolgus macaques infected with SIV is associated with early peripheral $\mathrm{T}$ cell depletion and not virus load. PLOS ONE 5, e9611 (2010).

10. Kalsdorf B, Scriba TJ, Wood K et al: HIV-1 infection impairs the bronchoalveolar T-cell response to mycobacteria. Am. J. Respir. Crit. Care Med. 180, 1262-1270 (2009).

11. Beveridge NE, Price DA, Casazza JP et al.: Immunisation with $\mathrm{BCG}$ and recombinant MVA85A induces long-lasting, polyfunctional Mycobacterium tuberculosisspecific $\mathrm{CD}^{+}{ }^{+}$memory $\mathrm{T}$ lymphocyte populations. Eur. J. Immunol. 37, 3089-3100 (2007). 
12. Lawn SD, Pisell TL, Hirsch CS, Wu M, Butera ST, Toossi Z: Anatomically compartmentalized human immunodeficiency virus replication in HLA-DR ${ }^{+}$cells and CD $14^{+}$macrophages at the site of pleural tuberculosis coinfection. J. Infect. Dis. 184, 1127-1133 (2001).

13. Lawn SD, Butera ST, Shinnick TM: Tuberculosis unleashed: the impact of human immunodeficiency virus infection on the host granulomatous response to Mycobacterium tuberculosis. Microbes Infect. 4, 635-646 (2002).

- Detailed review of the copathogenesis of TB and HIV-1 infection.

14. Sonnenberg P, Glynn JR, Fielding K, Murray J, Godfrey-Faussett P, Shearer S: How soon after infection with HIV does the risk of tuberculosis start to increase? A retrospective cohort study in South African gold miners. J. Infect. Dis. 191, 150-158 (2005).

- First study to document the early rise in risk of TB following HIV-1 seroconversion.

15. Glynn JR, Murray J, Bester A, Nelson G, Shearer S, Sonnenberg P: Effects of duration of HIV infection and secondary tuberculosis transmission on tuberculosis incidence in the South African gold mines. AIDS 22, 1859-1867 (2008).

16. Holmes CB, Wood R, Badri M et al.: CD4 decline and incidence of opportunistic infections in Cape Town, South Africa: implications for prophylaxis and treatment. J. Acquir. Immune Defic. Syndr. 42, 464-469 (2006).

17. Yazdanpanah Y, Chene G, Losina E et al.: Incidence of primary opportunistic infections in two human immunodeficiency virusinfected French clinical cohorts. Int. J. Epidemiol. 30, 864-871 (2001).

18. Antonucci G, Girardi E, Raviglione MC, Ippolito G: Risk factors for tuberculosis in HIV-infected persons. A prospective cohort study. The Gruppo Italiano di Studio Tubercolosi e AIDS (GISTA). JAMA 274, 143-148 (1995).
19. Markowitz N, Hansen NI, Hopewell PC et al.: Incidence of tuberculosis in the United States among HIV-infected persons. The Pulmonary Complications of HIV Infection Study Group. Ann. Intern. Med. 126, 123-132 (1997).

20. Wood R, Liang H, Wu H et al.: Changing prevalence of tuberculosis infection with increasing age in high-burden townships in South Africa. Int. J. Tuberc. Lung Dis. 14, 406-412 (2010).

21. Lawn SD, Bekker LG, Middelkoop K, Myer L, Wood R: Impact of HIV infection on the epidemiology of tuberculosis in a peri-urban community in South Africa: the need for age-specific interventions. Clin. Infect. Dis. 42, 1040-1047 (2006).

22. Lawn SD, Kranzer K, Wood R: Antiretroviral therapy for control of the $\mathrm{HIV}$-associated tuberculosis epidemic in resource-limited settings. Clin. Chest Med. 30, 685-699 (2009).

23. Lawn SD, Bekker LG, Wood R: How effectively does HAART restore immune responses to Mycobacterium tuberculosis? Implications for tuberculosis control. AIDS 19, 1113-1124 (2005)

24. Lawn SD, Myer L, Edwards D, Bekker LG, Wood R: Short-term and long-term risk of tuberculosis associated with CD4 cell recovery during antiretroviral therapy in South Africa. AIDS 23, 1717-1725 (2009).

25. Granich RM, Gilks CF, Dye C, De Cock KM, Williams BG: Universal voluntary HIV testing with immediate antiretroviral therapy as a strategy for elimination of HIV transmission: a mathematical model. Lancet 373, 48-57 (2009).

26. von Reyn CF, Mtei L, Arbeit RD et al.: Prevention of tuberculosis in Bacille Calmette-Guerin-primed, HIV-infected adults boosted with an inactivated whole-cell mycobacterial vaccine. AIDS 24, 675-685 (2010).
27. Akolo C, Adetifa I, Shepperd S, Volmink J: Treatment of latent tuberculosis infection in HIV-infected persons. Cochrane Database Syst. Rev. 1, CD000171 (2010).

28. Whalen CC, Johnson JL, Okwera A et al.: A trial of three regimens to prevent tuberculosis in Ugandan adults infected with the human immunodeficiency virus. Uganda-Case Western Reserve University Research Collaboration. N. Engl. J. Med. 337, 801-808 (1997).

29. Jasmer RM, Saukkonen JJ, Blumberg HM et al.: Short-course rifampin and pyrazinamide compared with isoniazid for latent tuberculosis infection: a multicenter clinical trial. Ann. Intern. Med. 137, 640-647 (2002).

30. Young DB, Gideon HP, Wilkinson RJ: Eliminating latent tuberculosis. Trends Microbiol. 17, 183-188 (2009).

31. Mtei L, Matee M, Herfort $\mathrm{O}$ et al.: High rates of clinical and subclinical tuberculosis among HIV-infected ambulatory subjects in Tanzania. Clin. Infect. Dis. 40, 1500-1507 (2005).

32. Dawson R, Masuka P, Edwards DJ et al.: Chest radiograph reading and recording system: evaluation for tuberculosis screening in patients with advanced HIV. Int. J. Tuberc. Lung Dis. 14, 52-58 (2010).

33. Wood R, Middelkoop K, Myer L et al: Undiagnosed tuberculosis in a community with high HIV-prevalence: implications for TB control. Am. J. Respir. Crit. Care Med 175, 87-93 (2007).

\section{Websites}

101. United Nations: The Millenium Development Goals Report 2008 www.un.org/millenniumgoals/

102. WHO: Global tuberculosis control. A short update to the 2009 report www.who.int/tb/publications/global_ report/2009/update/tbu_9.pdf

- Summary of the global burden of HIV-associated TB. 